Para enlazar con este artículo / To link to this article:

http://dx.doi.org/10.14198/fem.2017.29.08

Para citar este artículo / To cite this article:

Granada Angulo, Lubi. «Discriminaciones interseccionales: percepciones de mujeres afrodescendientes en espacios de educación superior en Bogotá». En Marcos Jesús Iglesias Martínez e Inés Lozano Cabezas (coords.), La (in) visibilidad de las mujeres en la Educación Superior: retos y desafios en la Academia. Feminismo/s, 29 (junio 2017): 201-220, DOI: 10.14198/fem.2017.29.08

\title{
DISCRIMINACIONES INTERSECCIONALES: PERCEPCIONES DE MUJERES AFRODESCENDIENTES EN ESPACIOS DE EDUCACIÓN SUPERIOR EN BOGOTÁ
}

\author{
INTERSECTIONAL DISCRIMINATIONS: PERCEPTIONS \\ OF AFRODESCENDING WOMEN IN SPACES OF HIGHER \\ EDUCATION IN BOGOTÁ
}

\author{
Lubi GRANADA ANGULO \\ Universidad Pedagógica Nacional \\ Secretaría de Educación Distrital - Bogotá (Colombia) \\ lubigra@yahoo.com \\ orcid.org/0000-0003-0144-9865
}

\section{Resumen}

El presente artículo forma parte de la etapa de exploración del proyecto de Tesis Doctoral de la autora. El propósito, que determina el ejercicio, se centra en la identificación de las percepciones sobre discriminación en espacios de Educación Superior en Bogotá (Colombia), que tienen tres mujeres afrodescendientes. Este trabajo se llevó a cabo utilizando la metodología de los Núcleos de Educación Social (NES), fortalecido a través de aportes teóricos y de investigación relacionados con el tema. Se evidencia de forma importante el peso de la discriminación negativa en la vida académica y laboral de las mujeres afrodescendientes participantes, que se ha constituido a lo largo del tiempo en una interseccionalidad entre categorías susceptibles de discriminación dentro y fuera de la Academia.

Palabras claves: discriminación negativa y positiva, Educación Superior, interseccionalidad, mujeres afrodescendientes. 


\begin{abstract}
This article is part of the exploration phase of the author's Doctoral Thesis project. The purpose of the exercise is to identify perceptions of discrimination in higher education spaces in Bogotá (Colombia), which have three women of African descent. This work was carried out using the methodology of the Nuclei of Social Education (NES), strengthened through theoretical and investigative contributions related to the topic. The weight of negative discrimination in the academic and work life of participating African descendants is evidenced, which has been constituted over time in intersectionality between categories susceptible of discrimination inside and outside the academy.
\end{abstract}

Keywords: negative and positive discrimination, higher education, intersectionality, women of African descent. 


\section{PUNTO DE PARTIDA}

Los estudios que sobre mujeres afrodescendientes se han adelantado en Colombia enmarcan una variedad de temáticas y problemáticas. De ellas resulta sugerente la recreación constante de la discriminación en diferentes discusiones académicas y sociales, a través de las cuales se posibilitan nuevas elaboraciones y preguntas para la reflexión.

En el marco de un proyecto de investigación doctoral ${ }^{1}$ surge como etapa preliminar la identificación de percepciones que sobre discriminación en espacios de Educación Superior tienen tres mujeres autorreconocidas como afrodescendientes, cuyas edades oscilan entre los 40 y 65 años de edad, con estudios de postgrado y trayectoria laboral en espacios de educación superior en Bogotá. Lo anterior con la idea de reconocer las diferentes facetas de la discriminación, así como sus intersecciones, dando cabida a la comprensión de complejas experiencias que sobre el tema se gestan.

A pesar de que este trabajo responde a un análisis que exige la ilación de tres aristas teóricas (mujeres afrodescendientes, Educación Superior y discriminación), para el presente ejercicio solo se retoma el eje de discriminación negativa y positiva desarrollado desde la perspectiva de Eduardo Restrepo, apoyado en aportes de otras y otros investigadores que sobre la materia han concentrado sus intereses, posibilitando así una triangulación con las percepciones de las mujeres afrodescendientes participantes.

Para identificar estas percepciones se emplearon los Núcleos de Educación Social (NES), teniendo en cuenta que es un modelo de investigación participativa, desarrollado a través de grupos (comunitarios, escolares, barriales, juveniles, estudiantes, entre otros), el cual permite conocer, indagar, interpretar, comprender, prevenir y transformar situaciones que pueden ser problemáticas en la realidad de los participantes que conforman los grupos de discusión (García y Guerrero 11).

1. El presente artículo expone reflexiones de una etapa de exploración correspondiente al proyecto doctoral titulado «Mujeres Afrodescendientes y Educación Superior: Estudio Interseccional y Multisituado en Colombia», que se está llevando a cabo en el Doctorado Interinstitucional en Educación, Sede Universidad Pedagógica Nacional (UPN, Bogotá). 
Los NES se construyen a través de 20 sesiones que se organizan en 4 escenarios $^{2}$ : el sujeto, la familia, la educación y los contextos; y 5 etapas ${ }^{3}$ : encuentros, exploratorios, recorridos, desplazamientos y transformaciones (García y Guerrero 37). Cada una de las sesiones del NES es organizada en una matriz con los elementos que se consideran necesarios para poner en evidencia la problemática de forma holística.

Las reflexiones de este artículo surgen a partir de los aportes realizados por tres mujeres afrodescendientes participantes en una sesión NES correspondiente a la etapa exploratoria en el escenario universidad, llevada a cabo en Bogotá, el día 23 de abril del año 2016; y se robustecen con planteamientos teóricos e investigativos que dan cuenta de la constante interseccionalidad entre raza y género en la percepción de discriminación mayoritariamente negativa que ellas identifican.

\section{A PROPÓSITO DE LOS ESPACIOS DE EDUCACIÓN SUPERIOR Y LAS MUJERES AFRODESCENDIENTES EN BOGOTÁ}

El nivel superior de la educación formal en Colombia presenta unas características que lo enmarcan en un sistema educativo consecuente con lineamientos, tiempos y estrategias que les permiten a las mujeres obtener conocimientos, habilidades y competencias para acceder a mejores oportunidades laborales, que en consecuencia traería un incremento en los niveles de calidad de vida. En este sentido, uno de sus tantos objetivos es la transformación y el desarrollo de sus vidas y de la sociedad (Mercado 9).

Sin embargo, el disfrute de esta posibilidad por parte de las mujeres afrodescendientes es vista más como una prerrogativa y no como el derecho del cual todas y todos los ciudadanos deben disfrutar: «Reconozco que es un privilegio llegar a un espacio universitario y encontrar con que el trabajo va a hacer dentro de lo que es relevar y no esconder mi identidad» (Mujer 01, NES Universidad, Sesión 12, 23 de abril de 2016).

Al retomar la participación de formación académica universitaria de las mujeres afrodescendientes en Bogotá, cabe referenciar el estudio titulado «Mujeres enseñando e investigando en la Universidad Nacional de Colombia»

2. Los escenarios corresponden a los lugares sobre los cuales las mujeres reflexionan, en este caso se seleccionó exclusivamente el escenario universidad, porque es el que compete para el presente ejercicio.

3. Corresponden a los momentos de reflexión y discusión que permiten los cambios y los tránsitos en las formas de percibir las problemáticas por parte de las mujeres. En esta oportunidad se desarrolló exclusivamente la etapa exploratoria considerando los intereses de la indagación. 
realizado en dicha institución en el año 2011, donde se menciona que más del $70 \%$ de los docentes son hombres y las mujeres partícipes, pertenecen a carreras consideradas femeninas, tales como humanidades, psicología y educación. Esta realidad tiende a repetirse en la mayoría de las universidades más importantes de Colombia y Latinoamérica. Además, las investigadoras plantean que esta situación es generada por diferentes factores, algunos asociados a la discriminación abierta y la falta de credibilidad entre pares (Munévar y Arana 14).

Con la investigación anterior, se puede establecer que la brecha de participación y liderazgo está presente en la realidad de la mayoría de las mujeres que en este campo se desenvuelven. Ahora bien, sugiere de manera implícita que las distancias en el acceso y participación para cargos de docencia, investigación y coordinación en espacios de Educación Superior para las mujeres afrodescendientes son aún más amplias. Tal como se evidencia en el siguiente relato:

Ahora llegando a la Universidad Nacional, yo fui la primera profesora negra del departamento de nutrición [...] eso fue un año antes de entrar en la convocatoria, porque estuve un tiempo ahí y yo era muy consciente. Lo que tú dices, a raíz de mi concientización en Gran Bretaña, yo llegaba a cualquier espacio y yo enseguida miraba a ver aquí quiénes son negros y siempre estábamos en minoría. (Mujer 02, NES Universidad, Sesión 12, 23 de abril de 2016)

En este sentido, establecer como punto de partida que la participación en los espacios de Educación Superior para mujeres afrodescendientes en Bogotá tiende al privilegio, implica reconocer también que en el disfrute de este derecho, se evidencian diferentes factores que obstruyen de alguna manera su máximo beneficio. Así pues, es importante delinear que dentro de los aspectos más representativos se encuentra la discriminación negativa, orientada con mayor fuerza a la categoría racial, pero haciendo fuertes lazos con el género y las habilidades cognitivas.

\section{ELEMENTOS PARA LA COMPRENSIÓN DE LA DISCRIMINACIÓN NEGATIVA}

Entender la discriminación negativa como un fenómeno que afecta a diferentes miembros de una comunidad, implica relacionar las dinámicas de diferenciación ${ }^{4}$ y exclusión que en ella se establecen. Las primeras, hacen referencia a las maneras de clasificación que se usan para distinguir entre las personas y los grupos. Por otro lado, la exclusión está relacionada con aquellas prácticas de

4. Las dinámicas de diferenciación también son empleadas como estrategias dentro de la discriminación positiva, aspecto que se ampliará más adelante. 
pensamiento o comportamiento que tienden al «rechazo, la negación y desconocimiento de quien es objeto de discriminación» (Restrepo s.f., 1).

De acuerdo con el planteamiento anterior, la diferenciación y la exclusión se convierten en las dos columnas en las que se erige la discriminación, propiciadas por cualquier marcador de diferencia. Como dinámica compleja, la discriminación se afianza con muchas posibilidades de acción en la realidad de las personas, retomando características como raza, género, clase o situación económica, educación, edad, estética, ubicación geográfica, elementos lingüísticos, religiosos, entre muchos otros. Así pues, las tipologías de discriminación son tan variadas como las características susceptibles de diferenciación, pero éstas, así como pueden revelarse de manera independiente, también consiguen relacionarse fuertemente entre ellas, tal como lo plantea Eduardo Restrepo:

No debe perderse de vista que la insistencia en distinguir entre diferentes tipos de discriminación dependiendo del anclaje concreto sobre el que se ejerce, no significa que estas discriminaciones se presenten de manera aislada. Al contrario, en muchas ocasiones nos encontramos con amalgamas de discriminaciones que se articulan y refuerzan mutuamente. Discriminaciones de orden racial se encuentran frecuentemente asociadas a las discriminaciones de clase, lingüísticas (formas de hablar) o geográficas (quienes son discriminados provienen generalmente de regiones marginalizadas y subalternizadas). Esto se debe a que los individuos y poblaciones concretos se encuentran atravesados al tiempo por muchas variables (raciales, sexuales, de clase, etc.) y algunas de éstas tienden a asociarse. (s.f., 2)

Lo anterior se conoce como interseccionalidad ${ }^{5}$, concepción que hace referencia al entrecruce de categorías que permite comprender cómo estas relaciones consolidan las experiencias de vida de las personas otorgando mayores elementos para la oportunidad o por el contrario, para la imposibilidad. Este elemento se convierte en herramienta crucial en la comprensión de las percepciones que las mujeres afrodescendientes tienen sobre la discriminación en los espacios de Educación Superior, pues están determinadas por al menos dos marcadores de

5. El concepto surge en Norteamérica con el artículo titulado: «Demarginalising the intersection of Race and Sex: A Black Feminist Critique of Antidiscrimination Doctrine, Feminist Theory and Antiracist Politics» (Crenshaw 1989). Sin embargo, éste se ha constituido y a la vez ha sido fundamento de las posturas críticas en América, permitiendo la comprensión del complejo entramado de las dinámicas y experiencias diversas de las mujeres en el continente. Desde este enfoque, se pueden reconocer aportes de los feminismos: negro, chicano y afrolatinoamericano (La Barbera 2014). Éstos en su conjunto aportan a la reflexión por medio del análisis de distintas categorías coloniales como los son la raza, la clase, el género, el sexo y la sexualidad principalmente, además de aportar en la comprensión de los efectos de estas variables, que pueden ser asumidas como estables y cambiantes en las sociedades (Curiel 2007, 96). 
diferencia (género y raza) que se relacionan entre sí y del cual emergen otras categorías susceptibles de discriminación como las referidas a las habilidades cognitivas.

\subsection{El racismo: la tipología de discriminación con mayor peso}

El racismo es el tipo de discriminación referido a la raza. Este elemento de análisis que surge en el proceso de colonización de América, y está relacionada con «la categorización de individuos según su nivel de similitud o cercanía respecto de un modelo presupuesto de humanidad ideal» (Mignolo 41).

Tal como lo propone Walter Mignolo, esta diferenciación se basa en una estructuración que ubica lo ideal blanco en el más alto nivel de jerarquía y a los diferentes en un nivel inferior. Este proceso se dio inicialmente a través de las religiones, aproximadamente para el siglo XVI, donde se hablaba principalmente de la "pureza de sangre», luego pasó a ser un proceso relacionado con «el color de piel» en el siglo XIX. Dentro de estos dos momentos de la constitución de la raza, se destaca el predominio de lo físico. Así pues, la raza está referida a «la genealogía sanguínea, genotípica o de color de piel» (Ver 41).

En este sentido, entender el racismo dentro de la lógica de superioridad de una raza sobre otra, implica establecer relaciones de tipo ideológico y práctico, que resuenan en la realidad cotidiana de las mujeres afrodescendientes. Tal como se evidencia en el siguiente apartado, en el que una de las mujeres participantes vincula de forma inmediata la raza con una tipología de discriminación negativa:

En el caso de nosotras como mujeres afro, eh, la discriminación es, lo primero que se presenta, es el color de la piel, -cierto-y entre ese color de la piel está la historia, la historia de la que no nos han reconocido a nuestros ancestros, que queda de los esclavos. (Mujer 01, NES Universidad, Sesión 12, 23 de abril de 2016)

De lo anterior, pareciera que existe una impronta racial difícil de modificar en las percepciones que sobre discriminación tienen las mujeres afrodescendientes entrevistadas. Esta situación puede deberse a la herencia de estigmatización que tiende a reproducirse en los discursos de las mujeres y la sociedad en general. Con esto se logra entender que la permanencia y cristalización de esta ideología se aferra al pensamiento social, se reproduce tanto en el discurso como en la práctica y permanece en los distintos escenarios donde circula. (Hellebrandová 88)

Para las sociedades donde se ha impuesto, manifestado y reproducido jerarquías raciales, resulta complejo desmontar este tipo de dinámicas, considerando que son procesos articulados al pensamiento colectivo que se perpetúan 
en los espacios simbólicos y físicos de la representación y la interacción social, a pesar de las modificaciones que se puedan hacer a las estructuras sociales. Al respecto, Lamus plantea que en Colombia existe una negación consciente del racismo, situación propiciada con el surgimiento del multiculturalismo, pues a través de éste se hace un reconocimiento a la diversidad cultural y se promueve la tolerancia entre los diferentes grupos étnicos. Sin embargo, dicha postura se convierte en una cortina que esconde en el fondo un entramado de discriminaciones basadas en la raza que no ha sido reconocido por la sociedad (163). Este aspecto se evidencia con claridad en la percepción de una de las mujeres:

Cuando yo con mis amigas muy cercanas... digo el racismo tal cosa, me dicen, pero es que no eres tan negra (risas)... Es que tú eres demasiado pasional, tú le estas poniendo mucha tiza a las cosas, ¿quién te discrimina a ti? (Mujer 02, NES Universidad, Sesión 12, 23 de abril de 2016)

Con relación al anterior relato, se puede vislumbrar uno de los elementos más peligros de la discriminación racial, referida la negación o la minimización de la misma, a través de la cual se genera una idea de exageración o victimización por parte de las personas que la perciben, haciendo que este proceso se convierta en un acto de responsabilidad exclusiva de la persona que se siente víctima. Al respecto, se puede afirmar que el racismo no ha desaparecido, sólo ha tomado otras formas y maneras de permanencia en las dinámicas sociales.

Siguiendo con esta idea y retomando los planteamientos de Eduardo Restrepo, se pueden establecer dos modos para ejercer el racismo: el primero, denominado «Manifiesto», en el que se evidencian explícitamente las dinámicas y las prácticas de discriminación a partir del color de piel o los rasgos fenotípicos; y el segundo designado como «Latente», es decir que se encuentra oculto, disfrazado y en muchas ocasiones naturalizado (6). Esta forma de ejercer el racismo, se gesta de manera inconsciente, pero resulta muy efectiva en la perpetuación de desventajas sociales:

La discriminación tiene muchas caras y formas de expresarse, no te podría decir con nombre propio, pero lo único que sí te puedo decir es que son muchas y de muchas formas, y las formas más peligrosas son las accesibles, invisibles y sutiles que no se dejan ver, pero están machacando todos los días lo que somos. (Mujer 03, NES Universidad, Sesión 12, 23 de abril de 2016)

Estas tipologías de racismo coinciden con lo que Hellebrandová presenta como racismo «solapado», del cual se niega su existencia, pues las prácticas discriminatorias no están abiertamente relacionadas al color de piel $(93)^{6}$. Según la

6. Al respecto, es pertinente resaltar que la categoría Raza ha estado relacionada fuertemente con la Etnia, pues esta última tiene que ver con las prácticas humanas que caracterizan 
investigadora, este tipo de prácticas son propias de las relaciones establecidas racialmente en América Latina, generando dificultades al enfrentar el racismo, pues no se evidencian explícitamente, por lo que parecieran inexistentes, pero forjan una «violencia latente» permeando y circulando entre las dinámicas institucionales. Por consiguiente, la discriminación racial tiene efectos evidentes o disimulados en la vida de las mujeres afrodescendientes, todos ellos establecidos en un marco institucional que posibilita su permanencia, prolongación y adecuación a los cambios sociales en tiempo y espacio. A esto se le conoce como «Racismo Estructural», definido exactamente como el

Diseño institucional que mantiene en la práctica la subalternización de unas poblaciones e individuos racialmente articulados. De ahí que esta dimensión del racismo atraviese todo el edificio institucional. Este racismo se encarna en acciones y omisiones concretas que, derivadas del funcionamiento mismo del sistema institucional, tienen el efecto de reproducir las desigualdades y jerarquías entre individuos y poblaciones racializadas. (Restrepo s.f., 7)

El racismo estructural justifica la exclusión y la invisibilización de las diferencias y necesidades de las comunidades racializadas. Esta situación se evidencia cuando institucionalmente se plantea que todas las personas están en las mismas condiciones para acceder a espacios de formación universitaria y las pone en el mismo lugar de competencia meritoria, desconociendo las desventajas acumulativas (en salud, educación, vivienda y acceso a servicios básicos) en las que sujetos y colectivos afrodescendientes se ven enfrentados de forma permanente.

Con este tipo de racismo se garantiza la materialización sistemática de marcos y escenarios geopolíticos, con los cuales se establecen relaciones entre los territorios, la raza y la etnia. Este aspecto se ve reflejado en normatividades como la Ley 70 de 1993, también conocida como ley de Comunidades Negras; en la que se reconoce la existencia de las poblaciones afrodescendientes, estableciendo una relación directa entre identidad étnica y territorio, referenciado específicamente a la región del Pacífico y algunos lugares del Atlántico

a los diferentes grupos que no son considerados dentro del supuesto del ideal humano. Retomando las palabras de Mignolo, la Etnia «incluye la lengua, la memoria y un conjunto de experiencias compartidas pasadas y presentes, por lo que comprende un sentido cultural de comunidad» (42). Este elemento es fundamental, pues ya no se estaría hablando exclusivamente de las características físicas, sino también de las actividades y prácticas relacionadas con lo físico. Así pues, hablar de afrodescendencia implica retomar tanto lo fenotípico (raza) y los elementos y prácticas culturales alrededor de estas caracterizaciones físicas (etnia). 
colombiano $^{7}$. De manera superficial este aspecto tiene efectos de reconocimiento de la diversidad en el país, pero al hacer un análisis de fondo muestra fuertes relaciones entre la distinción geopolítica racializada y las situaciones de precariedad y marginalidad de las personas que habitan en estos territorios ${ }^{8}$. Esta realidad se hace evidente en los planteamientos de una de las mujeres:

La discriminación es una cosa (...) que está anclada en las estructuras sociales y políticas de este país. Es decir, no es gratuitito que las poblaciones afro e indígenas estén confinadas a los márgenes o a los límites de nuestros territorios, casi que los lugares de exclusiones visibles para el país, donde las políticas llegan, pero llegan a medias o no llegan y es bastante complicado. Es decir, que desde que nos conformamos como Nación, ya nos cubrimos la espalda de los que teníamos poco o de los que somos considerados como menos. (Mujer 03, NES Universidad, Sesión 12, 23 de abril de 2016)

Como ya se ha dicho, la discriminación racial es evidente tanto en las prácticas cotidianas como en las institucionales, dejando a su paso una serie de estereotipos o ideas preconcebidas que tienden a homogenizar y exagerar representaciones que sobre las mujeres afrodescendientes se tienen. Estas preconcepciones están relacionadas con la sexualidad, la pobreza, la pereza y el folclor. Al respecto, diferentes estudios comprueban ${ }^{9}$ a partir de entrevistas, relatos e historias de vida, cómo las representaciones que se tienen de mujeres afrodescendientes están vinculadas a comportamientos de marcada disponibilidad sexual, tendencia a la fiesta, el baile y la alegría que se irradia en actividades folclóricas. Como ejemplo del planteamiento anterior, se expone el relato de una de las mujeres participantes en el NES:

El otro día fui a una presentación de los festivales, esa negrita que sacan en el canal de Barranquilla me emputa, discúlpenme. Su gran celebración, su gran aplauso, yo digo, pero es que ella lo que hace es preservar (...) todas esas imágenes (...) Esa propaganda del límpido no sé qué. Entonces, ese racismo en el cine, en las novelas, los roles, por ejemplo: esa mujer que hizo de la costeña y el cachaco, ella dijo, ¡nunca había roles para mí, todos eran de limpiadora!, ella rehusaba todos esos roles hasta que le sacaron un rol para ella. Entonces, toda esa parte del rol, del modelo, de la imagen que tanto las comunidades afrodescendientes, como los otros ven y ayudan a perpetuar. (Mujer 02, NES Universidad, Sesión 12, 23 de abril de 2016)

Sumado a esto, se evidencian imaginarios relacionados con la pereza, la irresponsabilidad, el abandono y la pobreza, dejando a los afrodescendientes

7. Luego con los documentos CONPES 2909 de 1997 y CONPES 3169 de 2002 se reconoce la presencia de la población afro en todo el territorio nacional.

8. Para ampliar ver (Mesa Nacional de Organizaciones Afrocolombianas 2012).

9. Tal es el caso de Lozano 2010; Morales 2012; Hellebrandová 2014; Meneses 2014. 
relegados a percepciones vinculadas con cuestiones de clase (Lamus 156), que desconocen las situaciones en las que se encuentran las mujeres afrodescendientes, tal como se muestra en el siguiente argumento:

Y llego acá, a la universidad (...) al espacio de los profesores y oigo una compañera mía que dice: no, es que yo tengo que buscar una persona para cuidar a mi niño y llega una mujer negra, pero entonces mi marido dice que las negras huelen a feo. Yo escuché ese comentario y mira, yo no (...) ¿cómo así que las negras huelen a feo? ¿Cómo es esto? Mira, me dio tanta rabia, porque indudablemente dije aquí hay discriminación, hay racismo (...) pero ¿por qué?, entonces yo misma empecé la discusión y todo eso (...) yo tenía tanta rabia que me fui para donde una compañera llorando. (Mujer 02, NES Universidad, Sesión 12, 23 de abril de 2016)

Las maneras de establecer las relaciones sociales a partir de la racialización y las implicaciones que ésta posee, dejan como efectos la trasformación del racismo y la prolongación de estereotipos racializados; pero también abren la posibilidad para reflexionar sobre los mecanismos de reacción por parte de las mujeres afrodescendientes, como es el caso del mestizaje y blanqueamiento.

En el marco del proceso de racialización surge la ideología racial del mestizaje que conserva estructuras jerarquizadas de la raza, donde la supremacía blanca se mantiene y establece patrones de lo apropiado, bueno y deseable en la sociedad colombiana. Al presentarse los valores blancos como ideales en las dinámicas sociales, se evidencian reacciones de mujeres afrodescendientes para acercarse a lo que estos preceptos plantean. A esto se le conoce como blanquedad o blanqueamiento (whiteness), entendido como «un proceso continuo e inacabado que dirige los cuerpos en direcciones específicas, determinando la manera como 'ocupan' el espacio». (Ahmed 2007, 155; en Hellebrandová 88)

El mestizaje y el blanqueamiento se presentan como acciones de respuesta y acomodación por parte de la comunidad afrodescendiente frente a dinámicas de jerarquización racial, que pueden estar representadas en negación de las raíces africanas; cambios físicos como cirugías estéticas para modificar rasgos, o estéticos como alisar o cambiar el patrón del cabello; cambios en la forma de hablar y expresarse; búsqueda de vinculación emocional exclusiva con personas no afrodescendientes; entre otros. Ahora bien, vale aclarar que estas tendencias no siempre son realizadas de forma intencional, de hecho, el blanqueamiento se instaura de forma sutil en los diferentes modos de subjetivación.

Debido a la maleabilidad y conveniencia tanto del mestizaje como del blanqueamiento, estas dinámicas resultan en procesos que dan cuenta de endorracismo, es decir, transformaciones en los que de manera inconsciente las mujeres empiezan a interiorizar y naturalizar las diferentes manifestaciones del racismo dejando muchas veces de ser ellas mismas, para querer ser otras: 
Porque yo he tenido, a diferencia de ustedes, yo he estado más blanqueada, he estado más con población blanca. Hay una diferencia, mi contacto con gentes, con comunidades negras ha sido muchísimo menor. Entonces, yo sí creo a veces, que uno encuentra personas negras totalmente blanqueadas, en sus actitudes, discriminando contra la misma gente negra. (Mujer 02, NES Universidad, Sesión 12, 23 de abril de 2016)

Con lo anterior, se entiende que el blanqueamiento posee un poder estratégico para «disimular» el racismo, convirtiéndose así en un artefacto que reproduce estereotipos racistas dentro de las mujeres afrodescendientes como grupo étnico racializado.

Ahora bien, hasta este momento el documento presenta algunos elementos relacionados con el racismo, que no son vinculados directamente con los espacios de Educación Superior, pero revelan matices que permean este contexto, pasando a otros niveles y estableciendo otro tipo de relaciones con categorías como el género.

\subsection{Discriminación de género: en relación persistente con la raza}

Las percepciones de discriminación, relacionadas con el género ${ }^{10}$, se cruzan con dos aspectos diferentes. El primero de ellos corresponde a la feminización o masculinización de las carreras universitarias, aspecto que ha ido cambiando paulatinamente con el tiempo, sin embargo en las historias académicas de las mujeres participantes, fue un elemento determinante, tal como se puede apreciar en el siguiente planteamiento:

En otros espacios universitarios, nos ha tocado dividir, sí, en una forma sutil o en una forma franca, en una forma abierta ¿no?, y es más en mi experiencia en los tiempos que me tocó estudiar. La verdad es que cuando estuve en Cali, no había mucho enfrentamiento, digamos frente a lo que era, ser mujer, por el contrario, era más que todo un enfrentamiento frente a mi identidad de género, porque (...) si las mujeres tenemos que estudiar trabajo social o enfermería, pero los hombres no podían estudiar trabajo social. (Mujer 01, NES Universidad, Sesión 12, 23 de abril de 2016)

Como segundo elemento, se vincula la segregación experimentada al interior de las aulas de clase en el rol de estudiantes, al sentir una profunda invisibilización

10. La categoría género hace referencia a un sistema impuesto en la colonización, a través del cual se establecen los roles y los comportamientos de los sujetos de acuerdo a su sexo. En esta perspectiva, los hombres y las mujeres se convierten en los dos grupos de referencia, estableciendo que son «diferentes pero complementados y se asume como grupos homogéneos y descontextualizados» (Curiel 2011, 10). En este sentido, los comportamientos son asignados y asumidos de acuerdo a las características físicas y biológicas de las personas (Mignolo 41). 
o exclusión, demeritando sus capacidades y habilidades cognitivas. Esta situación puede estar asociada a la conciencia colectiva oculta acerca de las desventajas acumulativas que históricamente han hecho parte de los procesos de la comunidad afrodescendiente. A continuación, se presenta una de las percepciones al respecto:

Claro, cuando llego a Bogotá y me enfrento a que el aula, en el aula por ejemplo soy la única mujer afro y me distinguía, verdad, (risas) desde luego, o me ignoraban (...) Es decir, si alzaba la mano porque yo conocía ese tema, entonces, lo miran a uno ¿será que sí?, o mejor pasan de largo la vista. (Mujer 01, NES Universidad, Sesión 12, 23 de abril de 2016)

Así pues, al chocar con este tipo de dinámicas en los espacios de formación, las mujeres encuentran como alternativa realizar un mayor sacrificio para demostrar sus capacidades, además de mejorar su estatus académico. Este aspecto tiene un gran impacto en las representaciones que se gestan sobre educación por parte de las generaciones venideras, al reconocer las desigualdades en oportunidades y el poco impacto que tiene para ellos adelantar procesos académicos que les permitan mejorar su calidad de vida, pues se crea una imagen en la que las mujeres afrodescendientes en comparación con las mujeres no afro, deben hacer doble esfuerzo para alcanzar las metas que se pretenden (Morales 75).

Bueno, la discriminación, yo sí quisiera opinar sobre cómo se siente, es bajarle y quitarle uno como mujeres afro, siempre la reserva de que ¿esta sí sabrá?, eh, o digamos, o hay que ponerle las cosas bien bajitas para que pueda entenderlas, entonces explican tres veces, mira, ven te explico, ven te ayudo. (Mujer 01, NES Universidad, Sesión 12, 23 de abril de 2016)

A veces con los mismos compañeros de espacio, suele pasar, también uno tiene que demostrar que es bueno para estar al nivel del otro compañero, que tiene igual una maestría como usted, que está en lo mismo que usted y sabe lo mismo (...) pero te miran como la profesora negrita, porque suele pasar como que ... ¿quién es la profesora?, uno se para y entonces, ¿esa es la profesora?, vamos a ver qué nos va a decir o con qué va a salir. (Mujer 03, NES Universidad, Sesión 12, 23 de abril de 2016)

Los relatos anteriores ponen en evidencia cómo estas mujeres afrodescendientes tienen una presión constante por cumplir con los requerimientos esperados o demostrar las capacidades que las hicieron estar en los lugares donde se encuentran; generando en ellas sensaciones de evaluación y contraste entre su etnicidad y sus capacidades cognitivas. Sin embargo, es posible que parte de esta percepción se deba a elementos que han afectado su estima como mujer profesional, este aspecto está mediado por los estereotipos racializados que tiene que romper permanentemente, para sentirse valoradas y respetadas dentro de los contextos académicos en Bogotá. 
Desde esta perspectiva, se establece aquí un fuerte vínculo con los procesos de discriminación pre-mercado y mercado, asociados a desventajas acumulativas que se encuentran ligadas a la categoría étnico-racial. La discriminación pre-mercado hace referencia a «las desigualdades previas: menor cobertura y calidad de la educación y servicios; brechas en el acceso a la educación universitaria; segregación espacial -ubicación en barrios y poblados rurales, etc.» (Lamus 157).

La discriminación de mercado consiste en un «trato diferencial para personas preparadas y aptas, pero discriminadas por características observables como raza, etnia y género» (Ver 157). Este último tipo de discriminación consiste en que mujeres con las competencias para desempeñarse en un empleo sean discriminadas de alguna forma, por ejemplo, recibiendo un pago inferior al de otras personas que están realizando la misma actividad:

En un momento determinado yo me enteré de que a estas personas les estaban pagando más que a mí. Yo dije ipero un momento, el trabajo es el mismo!, ¿por qué yo recibo menos dinero?, entonces yo hice toda una reclamación a mis jefes al respecto, les dije que me parecía el colmo, que era un abuso, que era discriminación, siendo el mismo trabajo ¿por qué razón yo debía tener menos, cuando yo cumplía con todos los requisitos?, entonces en la reclamación dicen que: no sé qué paso... en todo caso, de todas maneras, yo hice mi reclamo y fui consciente de esa discriminación, fue tan molesto. (Mujer 02, NES Universidad, Sesión 12, 23 de abril de 2016)

Con lo anterior, se pone en evidencia una percepción de discriminación a partir de la diferencia en remuneración económica, pero no es claro si ésta se da por su condición de género o raza dentro del contexto en el cual se encuentra. Este elemento se relaciona directamente con otros que ya se han planteado a lo largo del documento, referidos a la interseccionalidad, es decir al cruce y relación constante entre varias categorías, que resultan difíciles de separar, generando experiencias complejas situadas en momentos y lugares particulares.

De acuerdo con lo desarrollado hasta este punto, se entiende que la discriminación negativa tiene diferentes facetas que la hacen protagonista en las vidas de las mujeres afrodescendientes participantes del NES. Sin embargo, es pertinente rescatar un elemento importante en la evolución que ha tenido el tema, referido a la discriminación positiva, como alternativa para mejorar la calidad de vida de las personas que históricamente se han encontrado en desventaja con respecto a otros grupos poblacionales. 


\section{DISCRIMINACIÓN POSITIVA: MÁS QUE UNA APUESTA POLÍTICA DE ACCIONES AFIRMATIVAS}

La discriminación positiva es una apuesta que surge en las ciencias sociales para revertir de alguna forma los procesos de discriminación negativa realizados históricamente con grupos o poblaciones marginalizadas (Restrepo 2013, 8). En la discriminación positiva se retoman las mismas características o marcadores de diferencia susceptibles a la diferenciación y la exclusión, pero en este caso se convierten en elementos que posibilitan la inclusión y el reconocimiento. En Colombia, especialmente en Bogotá, la discriminación positiva para comunidades afrodescendientes se ha visto anclada a políticas de acción afirmativa ${ }^{11}$, considerándolas como:

Un conjunto de Políticas, directrices, programas, proyectos y medidas administrativas, que responden a los derechos y peticiones de la población afrodescendiente del Distrito, para garantizarles condiciones apropiadas de atención y acceso a sus servicios y programas, que no se fundamentan exclusivamente en su condición de población vulnerable, sino que responden a acciones basadas en criterios de razonabilidad histórica dirigidos a garantizar el acceso a mejores oportunidades de desarrollo económico, social y cultural, así como a promover su inclusión, mediante la definición de componentes de atención específica en su beneficio, que integren recursos, procedimientos, indicadores, cupos y porcentajes mínimos de participación, para el mejoramiento de la calidad de vida con fundamento en criterios concertados de aplicación gradual y complementaria. (Alcaldía Mayor de Bogotá 24)

De lo anterior, es importante aclarar que la construcción de esta política distrital fue realizada por la Alcaldía Mayor de Bogotá en cabeza de Luis Eduardo Garzón en el año 2006, con el acompañamiento y asesoría de grupos académicos y organizaciones de comunidades negras, protagonistas en la consolidación de estrategias para el reconocimiento diferencial de la comunidad afrodescendiente.

Este elemento resulta importante en la comprensión del enfoque de la discriminación positiva, pues no es el resultado de una decisión unilateral o exclusiva de las administraciones. Por el contrario, es una apuesta que posibilita dar voz y reconocimiento a la comunidad afectada, con el fin de promover una justicia reparativa o compensatoria (Martínez 211). Esto quiere decir, que existe una conciencia compartida de los problemas históricos de la comunidad

11. Las políticas de acción afirmativa no se establecen exclusivamente para afrodescendientes, también abarca diferentes sectores sociales: mujeres, LGBTI, en situación de discapacidad, grupos étnicos (indígenas, comunidad room) y en situación de vulnerabilidad (desplazados por la violencia). 
afro y la importancia de su participación en búsqueda de soluciones, de tal manera que se emplean estrategias «reestructuradoras que buscan la igualdad efectiva, la participación y la voz democrática sustantiva» (Forester 334). Ahora bien, vale aclarar que no es voluntad espontánea de la administración adelantar este tipo de normativas, por el contrario, es el producto de fuertes presiones por parte de la comunidad afrodescendiente, convirtiéndose este último elemento en «una de las condiciones fundamentales para la existencia de una política» (Hinestroza 13). Tal como lo pone en evidencia el discurso de una de las mujeres:

Hay unos que dicen que, ay que mira la discriminación positiva, cuando se hacen las políticas públicas, cierto y dicen entones, hay dos clases de discriminación, la discriminación positiva porque por eso es que tenemos unas leyes, hay una ley 70, existen unos artículos que predominan la atención a la población, conseguimos un fondo de becas en el Instituto de Crédito para la Educación de los Afrodescendientes, en fin. Entonces dicen: ipero esa es una discriminación positiva!, no, esos son derechos, sí, que estamos reclamando como parte de la reparación y así era que se estaba viendo dentro del artículo transitorio 55. Pero como existen unos poderes, van cambiando la cosa y lo hace siempre ver como si fueran dádivas, sí, y nosotras a veces nos comemos este cuentico de las dádivas. (Mujer 01, NES Universidad, Sesión 12, 23 de abril de 2016)

Al considerar el planteamiento anterior, es claro que las políticas de acciones afirmativas son un interesante vehículo para equiparar las oportunidades entre distintos grupos y colectividades. Pero no pueden ser éstas las únicas estrategias que permitan garantizar los derechos que como colombianas poseen. En otras palabras, la voluntad que se desprende de las acciones afirmativas es un valor agregado que debería generar mayores oportunidades en el acceso y la participación en espacios de Educación Superior, y no ser considerado como la única alternativa para acceder a un derecho que por obligación debe ser reconocido.

Después de todo, este tipo de lineamientos diferenciales en Bogotá emplean mecanismos de sistema de cuotas y trato preferencial para las mujeres afrodescendientes, que en muchas ocasiones quedan a la merced de la voluntad administrativa del momento. En este sentido, las acciones afirmativas son prácticas que pueden ser empleadas o no, en distintos escenarios y de maneras diversas y no siempre están amparadas por las políticas que las obligan. Pero, es necesario resaltar que la movilización y la organización de las comunidades afro generan nuevas posibilidades para que la aplicación de éstas sea efectiva:

Un día el secretario general de la universidad pasó y nos vio sentados en un espacio que se llama el malecón, nos ve, era todos los sábados y el compromiso era que teníamos que ir, esa no era una cosa de que un día iba u otro no, nada, 
ese era el compromiso. Él pasó, fue y nos preguntó, yo veo que los estudiantes negros, dijo él, los veo aquí sentados todos los sábados, ¿ustedes qué es lo que hacen?, entonces le contamos qué era lo que estábamos haciendo, por qué nos reunimos, entonces al señor le pareció genial la idea, y dijo: miren, yo puedo tomar una decisión aquí y la voy a tomar ahora. Todos los estudiantes que pertenecen a Afrocentral de ahora en adelante tienen el 30\% de descuento en su matrícula, pero tiene que demostrarme además que hacen un trabajo con el grupo, pero además el nivel académico también tiene que ser bueno. Es decir, que tienen un descuento en el porcentaje de la matricula porque está en Afrocentral y ya, tienen que demostrar que tienen trabajo con la comunidad y que tienen buenas calificaciones. (Mujer 03, NES Universidad, Sesión 12, 23 de abril de 2016)

De lo anterior, se infiere que la discriminación positiva orientada hacia políticas de acción afirmativa son apuestas para justificar las acciones reparativas para las comunidades afrodescendientes, las cuales parecieran no tener un nivel de obligatoriedad en las diferentes instituciones de la capital colombiana, por el contrario, quedan a la espera de la presión y la lucha de las comunidades discretamente beneficiadas. En este sentido, sería interesante ver cómo el cumplimiento de este tipo de políticas se hace visible en diferentes escenarios, pues la situación que se evidencia en Bogotá, es de un reducido porcentaje de mujeres afrodescendientes en las universidades públicas y privadas; además de una baja representación de maestras, investigadoras y directivas afrodescendientes en espacios de formación y liderazgo.

\section{IDEAS PARA CERRAR LA REFLEXIÓN}

A pesar de la promesa neoliberal donde la educación se convierte en la apuesta más segura para mejorar la calidad de vida, las mujeres afrodescendientes participantes perciben dicha propuesta como un privilegio que pocas pueden obtener y no como el derecho del cual deberían gozar todas y todos los colombianos. Aspecto, entre muchos otros, evidenciado por ellas en la baja representatividad de la población afrodescendiente en los espacios de Educación Superior del país.

El escrito plantea que la discriminación tiene dos connotaciones: una positiva y una negativa, sin embargo, es evidente que las mujeres afrodescendientes participantes la asumen fuertemente desde la segunda perspectiva. Es en este aspecto que la discriminación racial se vincula como el elemento de mayor peso en las dinámicas experimentadas por dichas mujeres. Situación que está anclada a procesos culturales de larga data, referidos específicamente a la invisibilización e inferiorización de las y los sujetos con características fenotípicas ligadas a la ascendencia africana esclavizada. Por tanto, tiende a estar supeditada a un cúmulo de desventajas acumulativas, que han sido parte tanto de 
sus comunidades como de sus historias de vida, marcadas principalmente por la racialización de sus potencialidades y capacidades.

Ahora bien, en la medida que el multiculturalismo como estrategia de armonización cultural se instala en las prácticas institucionales y en las relaciones sociales cotidianas, el racismo se alberga en distintos escenarios, se disfraza, se manifiesta a través de nuevas formas y pareciera inexistente. Sin embargo, sus efectos son impactantes y dejan como únicos testigos a las mujeres que los sufren. Ante esta situación las afrodescendientes no quedan impávidas, por el contrario, asumen actitudes que les permitan entrar en el juego de la dinámica multicultural, a través del mestizaje y el blanqueamiento, convirtiéndose este proceso en una maquinaria de doble filo que, por un lado, les permite acomodarse a las prácticas establecidas por la cultura hegemónica, pero que, por otro lado, terminan naturalizando y reproduciendo aquellas prácticas que las aqueja.

Esta permeabilización racializada se encuentra latente en los contextos académicos en los que ellas se desenvuelven. Aunque las prácticas con matices racistas son encubiertas, tienden a filtrarse a partir de relaciones constantes con los roles de género y las habilidades cognitivas que ellas tienen para el desarrollo de las actividades que emprenden. En esta perspectiva, los efectos se evidencian en la evaluación constante que perciben por parte de pares académicos y estudiantes, en la que parecieran estar sujetas a demostrar permanentemente las capacidades que les han permitido ubicarse en el rol de estudiantes o docentes, en los diferentes espacios de educación superior.

Ahora bien, las acciones reparadoras, pensadas desde la discriminación positiva, son un elemento importante en la dinámica de visibilización e inclusión de las mujeres afrodescendientes en las distintas instituciones. Empero y de acuerdo con las percepciones de las participantes, es claro que sus avances han sido tímidos y conservadores, aspecto que se evidencia en su limitado desarrollo. Además, pareciera que el derecho a la educación para este colectivo debe estar blindado por políticas de acciones afirmativas dependientes de la movilización de la comunidad afrodescendiente que la requiere.

A manera de cierre, resulta interesante ver cómo este tipo de exploraciones permiten descifrar y comprender las dinámicas de discriminación negativa y positiva en los espacios de educación superior desde las percepciones de mujeres afrodescendientes. Empero, también es claro que es una apuesta compleja que debe ser ampliada en posteriores oportunidades, pues los análisis que alrededor del tema se desarrollan, corresponden a entramados de distintas categorías que requieren de: macro comprensiones ancladas a elementos sociales, culturales e históricos de los contextos en los que cada una de las mujeres hace parte; comprensiones micro, es decir, desde las experiencias académicas 
particulares y múltiples de cada una de las mujeres afrodescendientes; y comprensiones meso, que retomen tanto las convergencias como las divergencias suscitadas entre lo macro y lo micro.

\section{REFERENCIAS BIBLIOGRÁFICAS}

Alcaldía Mayor de Bogotá. Política pública distrital y plan integral de acciones afirmativas para el reconocimiento de la diversidad cultural y la garantía de los derechos de los afrodescendientes. Bogotá, 2006.

Castellanos, Gabriela. «La categoría de género y la educación superior: Una mirada a América latina desde Colombia». La Manzana de la Discordia 6 (2011): 25-40.

Crenshaw, Kimberlé. «Demarginalizing the Intersection of Race and Sex: A Black Feminist Critique of Antidiscrimination Doctrine, Feminist Theory and Antiracist Politics». University of Chicago Forum (1989): 139-167.

Curiel, Ochy. «Crítica poscolonial desde las prácticas políticas del feminismo antirracista». Nómadas 26 (2007): 92-101.

Curiel, Ochy. Género, raza y sexualidad. Debates contemporáneos. s.f. <www.urosario.edu.co> urosario-files>, consultado el 30-03-2016.

Forester, John. «La racionalidad limitada y la política de salir del paso». La hechura de las políticas. Ed. Luis Aguilar Villanueva. México: Miguel Ángel Porrúa Librero-Editor, 1992, 315-340.

García, Andrés. «Políticas étnicas afrocolombianas en educación superior: dinámicas identitarias en la Universidad de Antioquia». Afrorreparaciones: Memorias de la esclavitud y justicia reparativa para negros, afrocolombianos y raizales. Bogotá: Ed. Universidad Nacional de Colombia, 2007, 661-689.

García, Bárbara y Javier Guerrero. Núcleos de educación social - NES. Bogotá: Universidad Distrital Francisco José de Caldas, 2012.

Hellebrandová, Klára. «Escapando a los estereotipos (sexuales) racializados: el caso de las personas afrodescendientes de clase media en Bogotá». Revista de Estudios Sociales 49 (2014): 87-100.

Hinestroza, Simón. «Impacto de la política pública de las acciones afirmativas para la población afrocolombiana en Bogotá D.C. En el marco de los derechos étnicos». Corporación Internacional para el desarrollo Educativo 16 (2015): 10-29.

La Barbera, María. Video El Enfoque de la Interseccionalidad: Evolución desafíos y perspectivas. «Espacios desiguales, fronteras invisibles». Ciclo de conferencias sobre interseccionalidad, inclusión social y equidad. En el marco del proyecto MISEAL. México: Universidad Autónoma de México. 2014. < https://www. youtube.com/watch?v=rwTe96gUqYQ>, consultado el 16-03-2016.

Lamus, Doris. «Negras, palenqueras y afrocartageneras. Construyendo un lugar contra la exclusión y la discriminación». Reflexión Política 23 (2010): 152-166. 
Lozano, Betty. «El feminismo no puede ser uno porque las mujeres somos diversas. Aportes a un feminismo negro decolonial desde la experiencia de las mujeres negras del Pacífico colombiano». La Manzana de la Discordia 2 (2010): 7-24.

Martínez, Rocío. «Acciones afirmativas para población afrocolombiana en Bogotá: historia de la formulación de una política pública». Estudios afrocolombianos hoy: aportes a un campo transdisciplinario. Ed. Eduardo Restrepo. Popayán: Editorial Universidad del Cauca, 2013, 207-226.

Meneses, Yeison. «Representaciones sociales sobre etnoeducación y cátedra de estudios afrocolombianos en la formación del profesorado». Enunciación 18 (2013): 45-63.

Meneses, Yeison. «Representaciones sociales sobre afrodescendientes: la aventura cultural, la violencia sexual-género y luchas multidimensionales». Memoria y sociedad 37 (2014): 76-92.

Mercado, Álvaro. «Políticas de acceso de la población vulnerable a la educación superior, una visión desde la experiencia de la Universidad del Magdalena». Clío América 15 (2014): 8-21.

Mesa Nacional de Organizaciones Afrocolombianas. Informe de la situación en derechos humanos de las mujeres afrocolombianas. Colombia: Fondo para la Sociedad Civil Colombiana, 2013.

Mignolo, Walter. La idea de América Latina. La herida colonial y la opción decolonial. Barcelona: Gedisa, 2007.

Morales, Erica. «Black Boundary Lines: Race, Class and Gender among Black Undergraduate Students». UCLA Electronic Theses and Dissertations. 2012. $<$ http://escholarship.org/uc/item/6jr3v45n>, consultado el 20-09-2015.

Munévar, Dora e Imelda Arana. «Mujeres enseñando e investigando en la Universidad Nacional de Colombia». Revista Interamericana de Estudios Feministas 1 (2011): 6-22.

Restrepo, Eduardo. «Acción afirmativa y afrodescendientes en Colombia». Estudios afrocolombianos hoy: aportes a un campo transdisciplinario. Ed. Eduardo Restrepo. Popayán: Universidad del Cauca, 2013, 249-265.

Restrepo, Eduardo. Racismo y Discriminación. s.f. <http://www.ram-wan.net/restrepo/documentos/racismo.pdf>, consultado el 7-02-2016.

Sesión NES No 12. Escenario Universidad. Mujeres Afrodescendientes. Bogotá. 23 de abril de 2016.

Quijano, Aníbal. Colonialidad del poder y clasificación social. Buenos Aires: CLACSO, 2014.

Viáfara, Carlos y Nini Serna. «Desigualdad de oportunidades educativas en la población de 15 a 29 años en Brasil y Colombia, según autoclasificación étnico -racial». Sociedad y Economía 29 (2015): 151-174. 